\title{
Near-infrared integral-field spectroscopy of the companion to GQ Lupi ${ }^{\star}$ (Research Note)
}

\author{
A. Seifahrt ${ }^{1,2}$, R. Neuhäuser ${ }^{2}$, and P. H. Hauschildt ${ }^{3}$ \\ 1 European Southern Observatory, Karl-Schwarzschild-Str. 2, 85748 Garching, Germany \\ e-mail: andreas.seifahrt@eso.org \\ 2 Astrophysikalisches Institut, Universität Jena, Schillergässchen 2-3, 07745 Jena, Germany \\ 3 Hamburger Sternwarte, Gojenbergsweg 112, 21029 Hamburg, Germany
}

Received 28 September 2006 / Accepted 7 December 2006

\section{ABSTRACT}

Context. The first substellar companion of possibly planetary mass around a normal star, GQ Lup, has been directly imaged (Neuhäuser et al. 2005, A\&A, 435, L13). Besides the unknown formation history, the mass of such an object is a criterion to decide about its true nature.

Aims. We aim to determine the physical properties of the GQ Lup companion - effective temperate $\left(T_{\text {eff }}\right)$ and surface gravity (log $\left.g\right)$, and thus its mass independently from evolutionary models.

Methods. We use the adaptive optics integral-field spectrograph SINFONI at the VLT for near-infrared spectroscopy from 1.1 to $2.5 \mu \mathrm{m}$ with a resolution of $R=2500-4000$. We compare these spectra with synthetic atmospheric models (GAIA v2.0 cond).

Results. From the complete set of spectra we find a consistent effective temperature and surface gravity of $T_{\text {eff }}=2650 \pm 100 \mathrm{~K}$ and $\log g=3.7 \pm 0.5$ dex. Combined with a slightly revised luminosity of $\log L / L_{\odot}=-2.25 \pm 0.24$ dex for the companion, we determine a radius of $R=3.50_{-1.03}^{+1.50} R_{\mathrm{Jup}}$ and thus a mass of $\sim 25 M_{\mathrm{Jup}}$. The uncertainty of this value is rather high. Due to the large uncertainty of the surface gravity, the mass could range from 4 to $155 M_{\mathrm{Jup}}$. By comparing the paramaters of the companion of GQ Lup to the ones of 2MASS J05352184-0546085, published by Stassun et al. (2006, Nature, 440, 311), we conclude that the companion to GQ Lup A has a mass lower than $36 \pm 3 M_{\text {Jup }}$.

Key words. stars: low-mass, brown dwarfs - planetary systems - techniques: spectroscopic

\section{Introduction}

Neuhäuser et al. (2005) and Mugrauer \& Neuhäuser (2005) presented a faint common proper motion companion to the $1 \pm 1 \mathrm{Myr}$ old T-Tauri star GQ Lup. Based on both, a low resolution NACO spectrum $(R \sim 700)$ in comparison to early GAIA model atmospheres (Brott \& Hauschildt 2005) and evolutionary models (Wuchterl 2005), the companion was found to have spectral type M9 to L4 and a mass of up to a few Jupiter masses.

The mass estimate was, however, model-dependent. Due to the young age of GQ Lup, the most widely used evolutionary models from the Tucson group (Burrows et al. 1993) and the Lyon group (Chabrier et al. 2000) for low mass objects are not applicable, see, e.g., Baraffe et al. (2002).

The direct determination of the mass from effective temperature, surface gravity and luminosity was hampered by the low signal-to-noise ratio and low resolution of the NACO $K$-band spectrum that yielded $T_{\text {eff }}$ and $\log g$ with wide error margins only. Moreover, the fit of the observed spectrum against a grid of synthetic spectra from GAIA dusty atmosphere models did not deliver consistent results for the $\mathrm{CO}$ band features and the overall slope of the spectrum (see e.g. Guenther et al. 2005).

It was thus desirable to obtain new near-infrared spectra with higher signal-to-noise ratio and higher resolution over a wider

$\star$ Based on observations collected at the European Southern Observatory, Chile, in programmes 275.C-5033(A) and 077.C-0264(A). spectral range. Because of the large magnitude difference between GQ Lup A and its companion and their small separation of $\sim 0.7^{\prime \prime}$ the use of adaptive optics systems was mandatory. Hence, we used the adaptive optics integral-field spectrograph SINFONI at the Very Large Telescope (VLT) that provides a spectral resolution of $R \sim 2500$ in the $J$-band and $R \sim 4000$ in the $H$ and $K$-band.

We present the observations and describe the data reduction in Sect. 2. The spectral synthesis is outlined in Sect. 3. We present the results of the fitting process in Sect. 4, where we end with conclusions and a short discussion about the nature of the GQ Lup companion.

\section{Observations and data reduction}

\subsection{Observations}

SINFONI (Spectrograph for INfrared Field Observations) is a combination of two instruments. First, a MACAO (MultiApplication Curvature Adaptive Optics) type curvature adaptive optics (AO) module with visual wavefront sensor and 60 actuators. Second, a mid-resolution near-infrared spectrograph with an integral field unit: SPIFFI (SPectrograph for Infrared Faint Field Imaging), see Eisenhauer et al. (2003) and Bonnet et al. (2004). The advantage of this instrument that combines the spatial resolving power of an $8 \mathrm{~m}$ telescope with a midresolution spectrograph is the absence of wavelength dependent 
slit losses that occur on normal spectrographs with a narrow entrance slit, especially when combined with AO.

The first observations of the GQ Lup companion have been carried out in $K$ band in the night of Sept. 16, 2005. The observations followed the standard scheme of sky nodding for background subtraction. Eight nodding cycles with a integration time of 300 s per frame where obtained. We have chosen the smallest pixel scale $(12.5 \times 25.0$ mas $)$ of the instrument, yielding a field of view of $0.8^{\prime \prime} \times 0.8^{\prime \prime}$ to optimally sample the diffraction limited core of the target PSF. The bright primary, GQ Lup A, was used as the AO guide star and was placed outside the FOV. The companion was centered in the field.

The DIMM ${ }^{1}$ seeing during the science observations was $0.8-1.1^{\prime \prime}$. The strehl ratio, computed on a short integration of GQ Lup A was $\sim 40 \%$ and more than $99 \%$ of the energy was encircled in a core of $\sim 200$ mas FWHM.

$H$ and $J$ band observations have been obtained in the nights of April 24 and September 18, 2006, respectively. Ten (nine) target-sky nodding cycles of 300 s exposure time have been taken in the $H$ and $J$ band, respectively. Five frames in each band had a sufficiently high signal-to-noise.

The DIMM seeing for the five useful $H$ band observations was $1.0-1.4^{\prime \prime}$. The strehl ratio, measured similarly as for the $K$ band, was $\sim 20 \%$ and more than $99 \%$ of the energy was encircled in a core of $\sim 250$ mas FWHM.

No PSF calibration was possible for the $J$ band since no observation of GQ Lup A was taken. In situ strehl computations on the companion are not reliable because of the background contamination from GQ Lup A. We therefore judge from the seeing during the $J$ band observations $\left(0.8-1.1^{\prime \prime}\right)$, the airmass and the performance of MACAO on a strehl of 5-10\%. The core size of the $99 \%$ quartile of the encircled energy is estimated to be of the order of 350 mas FWHM.

\subsection{Data reduction}

The data format of SINFONI is highly complex. The FOV of the instrument is sliced into 32 slitlets and remapped onto a pseudoslit and dispersed by a grating. The original FOV has to be reconstructed from this spectrum. The success of this process depends on the proper identification of the slitlet positions on the detector and the correction of nonlinear distortions.

The first step of the data reduction was thus performed by using the SINFONI data reduction pipeline version 1.3 offered by ESO (Jung et al. 2006). The reduction routines of this pipeline where developed by the SINFONI consortium (Abuter et al. 2006). In this first step the images are cleaned from bad pixels, flat fielded and wavelength calibrated. A distortion correction is applied and the the FOV of SINFONI is reconstructed. The output of this procedure is a $3 \mathrm{D}$ fits cube containing about 2000 images of the source in wavelength steps according to the chosen setting of the grating.

A mean along the wavelength axis of such a cube is an equivalent of a broad-band image in $J, H$, or $K$ band, respectively. In Fig. 1 we show such images for all three bands. The companion is well visible in the centre of each image. Depending on the different strehl ratios in each band, the target is still contaminated with light from the halo of GQ Lup A, whose core is outside the FOV. This contamination is spatially variable since the Airy pattern of the PSF of the primary is wavelength depended, even within a given band. This effect is strongest in the $K$ band.

\footnotetext{
${ }^{1}$ Seeing value in the optical $(\lambda \sim 500 \mathrm{~nm})$, measured at zenith.
}
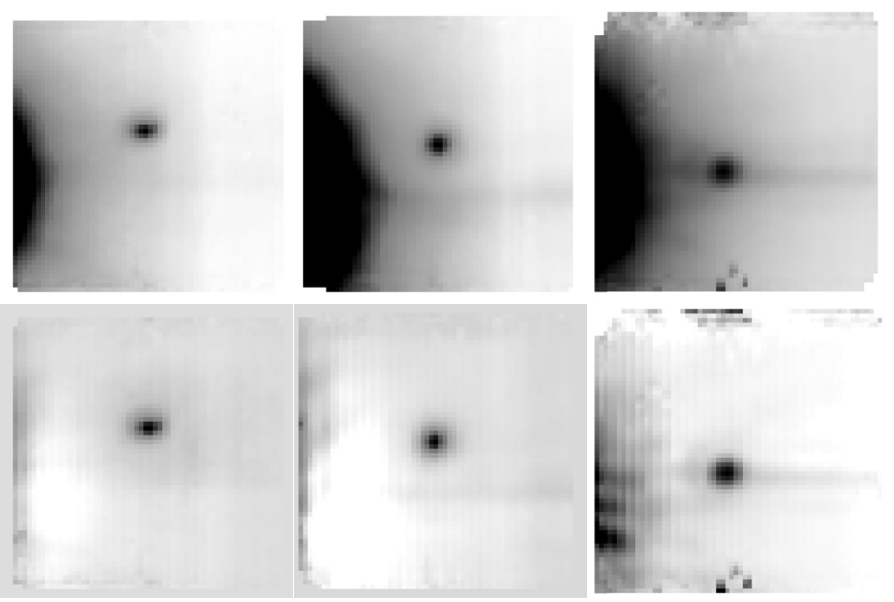

Fig. 1. From left to right: SINFONI $J, H$ and $K$ band cube of the GQ Lup companion, integrated over the the wavelength in each cube. Top row: before subtraction of the halo of GQ Lup A, bottom row: after subtraction. North is up and east is left. The FOV is $0.8^{\prime \prime} \times 0.8^{\prime \prime}$.

For a proper extraction of the target spectrum we have to eliminate this contamination as far as possible. We decided to use the Starfinder package of IDL (Diolaiti et al. 2000) for an empirical PSF fitting of the companion in each of the $2000 \mathrm{im}-$ ages of the three observed bands. For this purpose a template PSF is created from the observation of a standard star right before or after the target observation. The PSF of the standard star is thereby spatially supersampled from the $\sim 2000$ individual images of the source. The Starfinder algorithm determines the flux of the companion in each wavelength bin by fitting the previously created template PSF to the target. We found that one PSF template for each band is sufficient to ensure high correlation values (usually $\gtrsim 0.9$ ) in the fitting process, despite the fact that the PSF shape is variable over the covered band, as explained before. In an iterative process the smooth background from the PSF halo of GQ Lup A is subtracted as well. The lower column in Fig. 1 shows the images of the companion after this subtraction. The remaining speckles in the halo of GQ Lup A can not be filtered by this process. Since we fit a well sampled PSF to the target, the contribution from these localized remnants is, however, neglectable.

Eventually we obtained a spectrum of the GQ Lup companion from each nodding cycle in each of the three covered bands. These spectra are corrected for telluric absorption by the division through the spectrum of an early type telluric standard star. The following standard stars where chosen at the observatory to match the airmass of the science observations: HIP 087140 (B9V) for the $J$ band, HIP 082652 (B3III) for the $H$ band, and HIP 93193 (B9V) for the $K$ band. These stars are intrinsically featureless, apart from weak helium and strong hydrogen lines. These lines have been manually fitted and removed. The resulting spectrum was then multiplied by a blackbody spectrum to correct for the continuum slope of each standard star. The effective temperature of the blackbody was retrieved from the literature to $T_{\text {eff }}=10500 \mathrm{~K}$ for the stars of spectral type B9V and $T_{\text {eff }}=16500 \mathrm{~K}$ for the B3III type star, respectively. Note, that we are in the Rayleigh-Jeans regime of the standard stars SED. Hence, the steepness of the continuum slope is not particularly sensitive to small errors in the effective temperature. After this procedure the resulting spectra of the companion are essentially free of telluric absorption lines and corrected for the throughput of the spectrograph. 

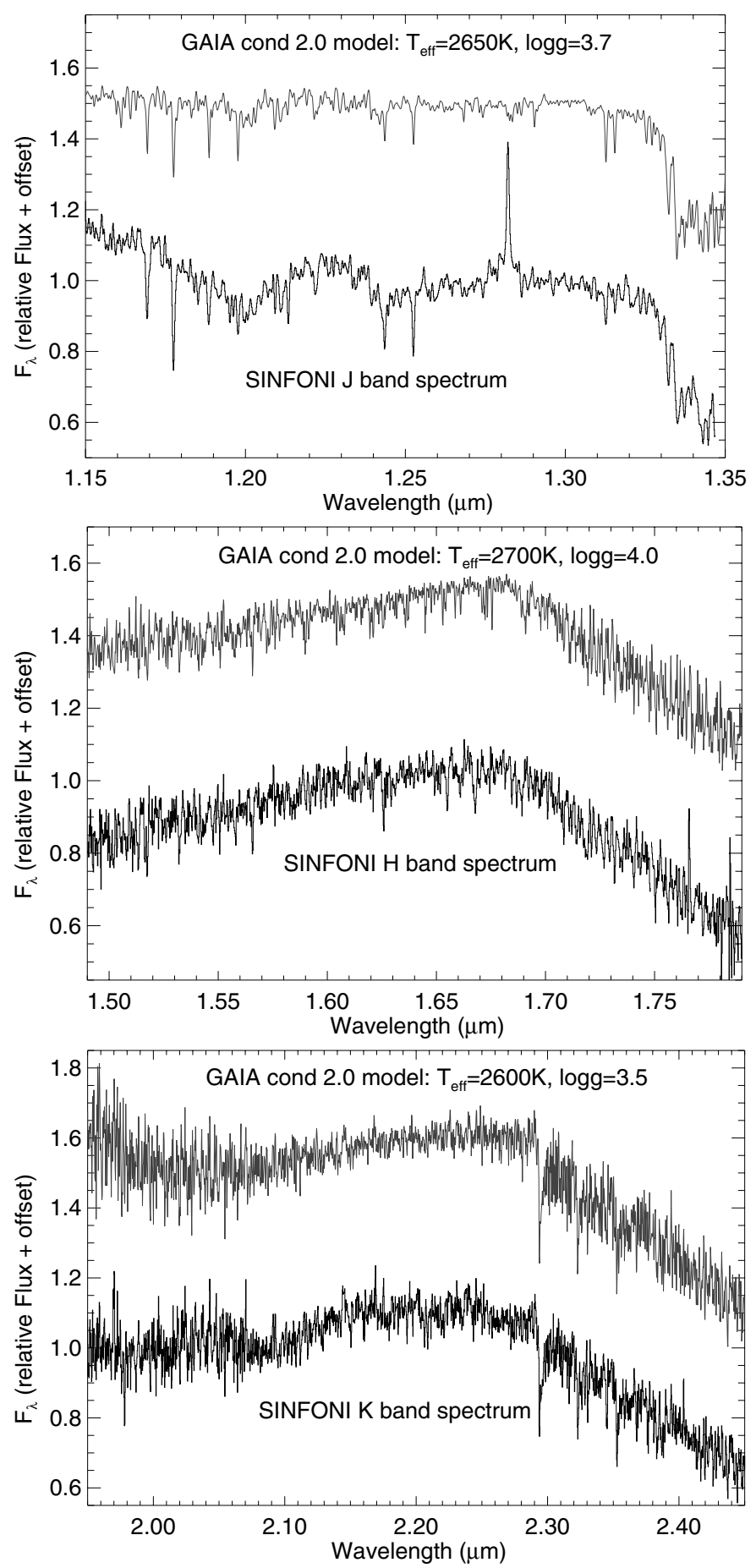

Fig. 2. From top to bottom: SINFONI $J, H$ and $K$ band spectra of the GQ Lup companion. The best fitting synthetic spectra are overplotted with a relative offset of +0.5 . The Nyquist sampled spectral resolution is $R \sim 2500$ in the $J$-band and $R \sim 4000$ in $H$ and $K$ band. Note the strong $\mathrm{Pa}-\beta$ emission line in the $J$-band.

The individual spectra from each nodding cycle are combined by a weighted mean. The weights are derived from the correlation factor of the PSF fitting. The three combined $J, H$ and $K$ band spectra are displayed in Fig. 2. The Nyquist sampled spectral resolution is $R \sim 2500$ in the $J$-band and $R \sim 4000$ in $H$ and $K$ band. Note, that the signal-to-noise ratio of the spectra is $\sim 100$ in the $J$ band but only $\sim 30$ in the $H$ and $K$ band. These values have been retrieved from the standard deviation in each spectral bin of the five ( $J$ and $H$ band) and eight ( $K$ band) individual spectra per band which have been reduced separately. The rather low signal-to-noise values origin from the spectral undersampling (1.5 pix per resolution element). Sub-pixel wavelength shifts between the individual nodding cycles induce artificial noise. However, most of the small-scale features seen in the spectra are not noise but unresolved absorption lines.

\section{Spectral synthesis}

We compared our observed spectra with the theoretical template spectra from the GAIA-Cond model v2.0 (Brott \& Hauschildt 2005), updated from Allard et al. (2001), with improved molecular dissociation constants, more dust species with opacities, spherical symmetry, and a mixing length parameter $2.0 \cdot H_{\mathrm{p}}$. The grid spans a range of $1000 \ldots 3000 \mathrm{~K}$ in effective temperature and $-0.5 \ldots 6.0 \mathrm{dex}$ in surface gravity. The metallicity is fixed at $[\mathrm{Fe} / \mathrm{H}]=0.0$. The stepsize of the grid is $100 \mathrm{~K}$ in effective temperature and 0.5 dex in surface gravity. Where appropriate we linearly interpolated the spectral grid to refine the fits to the measured spectra. At around $2600-2700 \mathrm{~K}$ (the final effective temperature we derived for the companion to GQ Lup - see below), the effect of dust on the spectra is still small (it starts to affect the spectra massively around $2400 \mathrm{~K}$ ). Furthermore, dusty models of this class were not available, thus we feel justified to use the cond models. The synthetic spectra are computed for a resolution of 200000 . We convolved the spectra with a Gaussian kernel to reduce the resolution to the one of the SINFONI spectra. Finally the synthetic spectra where re-binned to match the spectral sampling of the measured spectra. For the entire grid of synthetic spectra a $\chi^{2}$-algorithm was applied to determine the best-fitting model spectrum, see Fig. 2.

\subsection{Spectral features}

$J$ band

A water vapor band absorption longwards of $1.30 \mu \mathrm{m}$ is very well fitted by the models and gives strong constraint on the effective temperature. The strong metal lines (K I doublets at 1.169 and $1.178 \mu \mathrm{m}$ and at 1.244 and $1.256 \mu \mathrm{m}$ as well as Al I features at 1.313 and $1.315 \mu \mathrm{m}$ ) are very sensitive on the effective temperature and the surface gravity. However, their relative linestrengths can not be modeled accurately since the model grid has a fixed metallicity at $[\mathrm{Fe} / \mathrm{H}]=0.0$. Hence, these features have been left out of the fitting process. The most prominent feature is the $\mathrm{Pa} \beta$ emission line at $1.282 \mu \mathrm{m}$. This line shows an inverse P-Cygni profile in GQ Lup A. We identify the emission as being most likely caused by accretion in both objects.

The continuum of the $J$ band shortwards of $1.25 \mu \mathrm{m}$ looks exceptionally bumpy and is only marginally fitted by the models. The steep decrease in flux from 1.15 to $1.2 \mu \mathrm{m}$ is also seen in the spectra of the young field L dwarf, 2MASS J0141-4633, and most likely due to $\mathrm{VO}$ and $\mathrm{FeH}$ absorption (Kirkpatrick et al. 2006). However, in our spectrum it falls in a region that is most subject to remaining contamination from GQ Lup A. We thus decided to restrict the fitting process to wavelength longer than $1.2 \mu \mathrm{m}$. Moreover, the increasing slope from 1.20 to $1.23 \mu \mathrm{m}$ observed in the GQ Lup companion was also observed in 2MASS J0141-4633 and other young, low-gravity objects like KPNO-Tau 4 and G 196-3 B (McGovern et al. 2004) and is due to $\mathrm{FeH}$ bands. It is also not yet reproduced by our GAIA-cond model and has been consequently disgarded from the fit. 


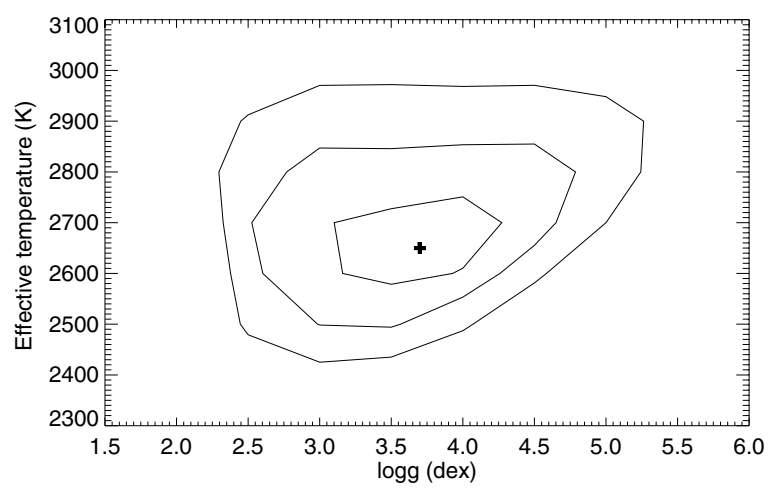

Fig. 3. Results from the fit of GAIA cond synthetic model spectra to the SINFONI $J, H$ and $K$ band spectra of the GQ Lup companion. Best value marked with a cross. Contours relate to 1,2 and $3 \sigma$ errors.

\section{$H$ band}

The continuum slope of the $H$ band exhibits a strong triangular shape that is usually identified with low-gravity objects. The nature of this feature is supposedly the $\mathrm{H}_{2}$ collision induced absorption (CIA), as discussed by Kirkpatrick et al. (2006, see references therein). The $H$ band is essentially free of strong absoption lines from molecules and metals. The constraints on the effective temperature and surface gravity is the lowest among the three bands.

\section{$K$ band}

The $K$ band spectrum shows an excellent agreement between model and measurement, both, in the slope of the pseudocontinuum and the depth of the most prominent spectral features. Again, water vapor absorption at both edges of the spectrum is a good indicator for the effective temperature. The ${ }^{12} \mathrm{CO}$ band heads longward of $2.29 \mu \mathrm{m}$ are also very well fitted. We identify a weak Na I doublet at $2.201 \mu \mathrm{m}$ which is however slightly better fitted by a $\log g$ determined from $J$ and $H$ band. We note a non-detection of $\mathrm{Br} \gamma$ line at $2.166 \mu \mathrm{m}$. This line is in emission in the spectra of GQ Lup A where it shows a slightly asymmetric profile.

\subsection{Determination of $T_{\text {eff }}$ and $\log g$}

The results of the fitting process are displayed in Fig. 3 for the combined $\chi^{2}$ minimisation in a $\log g-T_{\text {eff }}$ plane. The derived uncertainties (displayed in Fig. 3 as contours of 1,2 and $3 \sigma$ ) stand for the systematic errors. We used a Monte-Carlo simulation technique to infer the statistical errors on the determination of the model parameters. The uncertainties from the noise in the spectra where found to be much lower than the uncertainties from fitting errors. This demonstrates that the slope of the continuum is the major contraint as long as the spectral lines are not well resolved.

While the best fit for the $J$ band alone is $T_{\text {eff }}=2650 \mathrm{~K}$ and $\log g=3.70$ dex, the best fit for the $H$ and $K$ band spectrum have slightly different effective temperatures and surface gravities, as can be seen in Fig. 2. Since the $J$ band spectrum has the steepest $\chi^{2}$ curve, it is dominating the final solution, which we determine to be $T_{\text {eff }}=2650 \pm 100 \mathrm{~K}$ and $\log g=3.70 \pm 0.5$ dex.
Table 1. Comparing the GQ Lup companion to 2M0535 A and B.

\begin{tabular}{lccc}
\hline \hline Parameter & GQ Lup b/B $^{a}$ & 2M0535 A & 2M0535 B \\
\hline$T_{\text {eff }}[\mathrm{K}]$ & $2650 \pm 100$ & $2650 \pm 100^{b}$ & $2790 \pm 105^{b}$ \\
$\log \left(L_{\mathrm{bol}} / L_{\odot}\right)$ & $-2.25 \pm 0.24$ & $-1.70 \pm 0.11$ & $-1.74 \pm 0.11$ \\
Age $[\mathrm{Myr}]$ & $0-2$ & $0-3$ & $0-3$ \\
Radius $\left[R_{\odot}\right]$ & $0.36_{-0.11}^{+0.15}$ & $0.669 \pm 0.034$ & $0.511 \pm 0.026$ \\
Mass $\left[M_{\odot}\right]$ & $0.025_{-0.021}^{+0.120 c}$ & $0.054 \pm 0.005$ & $0.034 \pm 0.003$ \\
\hline
\end{tabular}

Remarks: ${ }^{a}$ GQ Lup b, if planet or planet candidate; GQ Lup B, if brown dwarf or star. ${ }^{b} \pm 300 \mathrm{~K}$ systematic uncertainty (2006). ${ }^{c}$ Regarding the upper limit: our companion is fainter and smaller than both $2 \mathrm{M} 0535 \mathrm{~A}$ and $\mathrm{B}$, hence lower in mass than the components of $2 \mathrm{M} 0535$.

\section{Results and discussion}

To determine the mass of the GQ Lup companion independently from evolutionary models, we need its surface gravity and radius. The latter can be derived from a previously determined $K$ band magnitude $K_{\mathrm{s}}=13.1 \pm 0.2 \mathrm{mag}$ (Neuhäuser et al. 2005) and a bolometric correction. Based on our new results for the effective temperature, we have to slightly revise the bolometric correction used in (Neuhäuser et al. 2005). From Table 6 of Golimowski et al. (2004) we determine $B C_{\mathrm{K}}=3.00 \pm 0.07 \mathrm{mag}$, interpolating over the objects with comparable effective temperature.

With these values, we derive a luminosity of $\log \left(L / L_{\odot}\right)=$ $-2.25 \pm 0.24$, slightly higher than in (Neuhäuser et al. 2005), but consistent within the error margin. From luminosity and temperature, we can then estimate the radius of the companion to be $R=3.50_{-1.03}^{+1.50} R_{\text {Jup }}$. Gravity and radius then determine the mass, for which we get $\sim 25 M_{\text {Jup }}$, however, with large uncertainty. The mass can be as low as $\sim 4 M_{\text {Jup }}$ for lower gravity or even as high as $\sim 155 M_{\text {Jup }}$ given the large uncertainty in the surface gravity.

At face value, the mass does not seem to be better contrained now, compared with Neuhäuser et al. (2005), who gave 1 to 42 Jupiter masses. However, we think that due to the low resolution, low signal-to-noise, and limited spectral coverage the surface gravity and effective temperature may have been underestimated in Neuhäuser et al. (2005). Even though our mass determination is independent from evolutionary models, it still depends on the atmospheric models. Until the GAIA model is tested and confirmed in the very low-mass and young age regime, our results are to be seen uncertain. However, we argue that the accuracy of a mass determined from spectral synthesis should be higher than from evolutionary models since the latter should use synthetic atmospheric models itself as an input parameter.

Stassun et al. (2006) found an eclipsing double-lined spectroscopic binary, 2MASS J05352184-0546085 (hereafter 2M0535), consisting of two brown dwarfs in the Orion star forming region. Their masses could be determined dynamically, hence independently from evolutionary models and model atmospheres. This makes 2M0535 most suitable for a direct comparison with the GQ Lup companion.

Note, that the more massive object in 2M0535 is, however, cooler, which is not expected from standard models (e.g. Lyon or Tucson groups). Stassun et al. (2006) also note a systematic uncertainty of $\pm 300 \mathrm{~K}$ in the absolute temperatures, and can give only a precise temperature difference or fraction.

We note that the companion to GQ Lup has roughly the same age but is fainter and smaller than both components in 2M0535 (Stassun et al. 2006), see Table 1. The effective temperature is comparable with the cooler component in this double. Judging 
from the temperature and radius of the nearly coeval objects, the GQ Lup companion must be lower in mass than each of the components in $2 \mathrm{M} 0535$, i.e. $\leq 36 M_{\text {Jup }}$.

Finally we note two recent results on GQ Lup:

Marois et al. (2006) fit the RIJHKL-band spectral energy distribution of the GQ Lup companion with a GAIA model to obtain physical parameters. They find a radius of $0.38 \pm 0.05 R_{\odot}$, and an effective temperature of $2335 \pm 100 \mathrm{~K}$. Hence, the authors confirm temperature and luminosity of (Neuhäuser et al. 2005). Their modelfits are reported to be gravity insensitiv and a $\log g$ of $3-4$ is assumed. They use the evolutionary models from the Lyon group to obtain a mass of 10 to 20 Jupitermasses.

McElwain et al. (2006) obtained an integral field $J$ and $H$-band spectrum with OSIRIS at Keck, which is lower in dynamic range, resolution, $\mathrm{S} / \mathrm{N}$, and spectral coverage than ours. They confirm the luminosity, and temperature from Neuhäuser et al. 2005 and obtain a mass of 10 to $40 M_{\text {Jup }}$.

Both authors use the models of the Lyon group. We argue that these models are not applicable for objects up to $10 \mathrm{Myr}$, following the discussion in Chabrier et al. (2005).

Based on our and otherwise published results, one still cannot decide, whether the companion to GQ Lup is a massive planet or a low-mass brown dwarf.

Acknowledgements. We would like to thank the staff of the VLT at Paranal for the execution of the observations. We also thank Eike W. Guenther for useful discussions about the layout of the measurement. Finally we thank the anonymous referee for the thorough review and the helpful comments.

\section{References}

Abuter, R., Schreiber, J., Eisenhauer, F., et al. 2006, New Astro. Rev., 50, 398 Allard, F., Hauschildt, P. H., Alexander, D. R., Tamanai, A., \& Schweitzer, A. 2001, ApJ, 556, 357

Baraffe, I., Chabrier, G., Allard, F., \& Hauschildt, P. H. 2002, A\&A, 382, 563

Bonnet, H., et al. 2004, The Messenger, 117, 17

Brott, I., \& Hauschildt, P. H. 2005, ESA SP-576: The Three-Dimensional Universe with Gaia, 565

Burrows, A., Hubbard, W. B., Saumon, D., \& Lunine, J. I. 1993, ApJ, 406, 158

Chabrier, G., Baraffe, I., Allard, F., \& Hauschildt, P. 2000, ApJ, 542, 464

Chabrier, G., Baraffe, I., Allard, F., \& Hauschildt, P. 2005, review, in press, [astro-ph/0509798]

Diolaiti, E., Bendinelli, O., Bonaccini, D., Close, L. M., Currie, D. G., \& Parmeggiani, G. 2000, Proc. SPIE, 4007, 879

Eisenhauer, F., et al. 2003, Proc. SPIE, 4841, 1548

Golimowski, D. A., et al. 2004, AJ, 127, 3516

Guenther, E. W., Neuhäuser, R., Wuchterl, G., Mugrauer, M., Bedalov, A., \& Hauschildt, P. H. 2005, Astron. Nachr., 326, 958

Guenther, E. W. 2006, Rev. Mod. Astron., 19, 237

Jung, Y., Lundin, L. K., Modigliani, A., Dobrzycka, D., \& Hummel, W. 2006, ASP Conf. Ser., 351, 295

Kirkpatrick, J. D., Barman, T. S., Burgasser, A. J., et al. 2006, ApJ, 639, 1120

Marois, C., Macintosh, B., \& Barman, T. 2006, ApJ, accepted [astro-ph/0610058]

McElwain, M. W., Metchev, S. A., Larkin, J. E., et al., 2006 , [astro-ph/0610265]

McGovern, M. R., Kirkpatrick, J. D., McLean, I. S., Burgasser, A. J., Prato, L., \& Lowrance, P. J. 2004, ApJ, 600, 1020

Mugrauer, M., \& Neuhäuser, R. 2005, Astron. Nachr., 326, 701

Neuhäuser, R., Guenther, E. W., Wuchterl, G., et al. 2005, A\&A, 435, L13

Neuhaeuser, R., Mugrauer, M., \& Seifahrt, A. 2006, IAU Symp., 240

Stassun, K. G., Mathieu, R. D., \& Valenti, J. A. 2006, Nature, 440, 311

Wuchterl, G. 2005, Astron. Nachr., 326, 905 